\title{
ADDRESSING DISADVANTAGE AND HEALTH: THE MOUNT DRUITT PILOT STUDY
}

\section{Ann Gethin \\ Population Health Partnerships Unit \\ University of Western Sydney}

The question of how to ameliorate health inequities is one of the main challenges facing the public health workforce. How might differences in health be addressed, which have their roots in poverty and social exclusion? What training do public health workers need to design effective programs and interventions for disadvantaged populations? Commissioned by a cross-institutional group; produced with funding from the Commonwealth Department of Health and Aged Care; and administered by the Centre for Health Equity Training and Research Evaluation, South Western Area Health Service, and the Population Health Partnerships Unit, University of Western Sydney, the Mount Druitt Pilot Study makes a small step towards answering these questions. This article introduces the study.

This qualitative study interviewed 22 people working on existing or proposed interventions in the Mount Druitt and surrounding area, which is one of the most highly disadvantaged areas in New South Wales. Seven workers were from health-related services, while fifteen workers were from a range of government, non-government, and community organisations. Workers from outside the health sector were included in the study, because many of the factors influencing health-for example, housing, crime, literacy, and social integration-are the direct concern of other agencies.

Participants were asked to identify:

- characteristics of projects that they considered could effectively address social disadvantage;

- barriers to successfully addressing social disadvantage;

- possible future directions for addressing the impact of social disadvantage on health.

Common themes were identified and grouped according to the above headings.

Interventions were considered a success if they had met their objectives; no interventions had been evaluated in the long term. Factors that the participants considered had contributed to success included: 'adequate funding', 'organisation support', and 'sufficient time-lines'. Less quantifiable factors were also identified, such as 'personality of key workers', 'good partnerships', and 'trust'. Although these factors are phenomena of which most community level workers are aware, it is a challenge to 'operationalise' them so that they might become part of a training program for health workers.

Trust, for example, appeared to be a function of cultural sensitivity, of the length of time a worker spends in the community, and workers not adopting patronising behaviours. These factors can be easily undermined by other forces often beyond the individual workers' control; for instance, in Mount Druitt the terms and conditions of work have led to a constant stream of health and welfare workers passing through the community. This loss of continuity erodes trust.

Although numerous examples of successful interventions were cited, a number of interviewees raised the issue of creating lasting improvements in social wellbeing and population health. Mount Druitt has been the target of a plethora of interventions over recent decades but the problems of poverty, poor health, addiction, and violence are enduring. Small-scale interventions were not seen as being able to address the deeper causes of disadvantage. For this reason, there is a strong impetus to begin integrating interventions and to look at more radical solutions. For example, public housing, which represents 30 per cent of housing in Mount Druitt, has high concentrations of the most socially and economically disadvantaged populations; consequently, the NSW Department of Housing is considering interventions aimed at changing the social and economic mix of residents living in public housing.

Poverty, and the accompanying health disadvantages, is becoming increasingly concentrated in particular areasa consequence of an entrenched income divide, declining housing affordability, and the legacy of large-scale public housing estates. The future public health workforce will require the skills and knowledge to work effectively with these communities.

The Mount Druitt Pilot Study is to be published as a monograph later this year. Further details can be obtained from the author by email at a.gethin@uws.edu.au. 